Enfants manifestant un trouble des conduites et utilisant des services psychoéducatifs : un portrait social, familial et psychologique

\author{
Children with conducts disorder and using psychoeducational \\ services: a social, psychological and family portrait
}

Los niños que manifiestan un problema de conducta y utilizan los servicios psicoeducativos: un retrato social, familiar y psicológico Retrato social, familiar e psicológico das crianças que manifestam problemas de comportamento e utilizam serviços psico-educativos

\author{
Jean Toupin, Robert Pauzé, Éric Yergeau, Michèle Déry, Laurier Fortin et Henri \\ Mercier
}

Volume 28, numéro 1, printemps 2003

De « meilleures pratiques " à « pratiques novatrices "

URI : https://id.erudit.org/iderudit/006990ar

DOI : https://doi.org/10.7202/006990ar

Aller au sommaire du numéro

Éditeur(s)

Revue Santé mentale au Québec

ISSN

0383-6320 (imprimé)

1708-3923 (numérique)

Découvrir la revue

Citer cet article

Toupin, J., Pauzé, R., Yergeau, É., Déry, M., Fortin, L. \& Mercier, H. (2003). Enfants manifestant un trouble des conduites et utilisant des services psychoéducatifs : un portrait social, familial et psychologique. Santé mentale au Québec, 28(1), 232-257. https://doi.org/10.7202/006990ar
Résumé de l'article

Les études contemporaines suggèrent que la plupart des enfants qui manifestent un trouble des conduites présentent également des difficultés personnelles et familiales. Ceci est particulièrement vrai pour les jeunes dont le trouble est précoce. L'identification des caractéristiques personnelles et des facteurs sociaux et familiaux de risque et de protection associés aux difficultés sont d'une grande utilité pour la planification des interventions et l'organisation des services à offrir aux jeunes et aux familles du Québec. En conséquence, les objectifs de cette étude sont d'identifier les facteurs sociaux, familiaux et psychologiques qui caractérisent les enfants présentant un trouble des conduites. Pour y parvenir 62 enfants en traitement, âgés entre 7 et 12 ans et présentant un diagnostic de trouble des conduites sont comparés à 36 enfants du même âge sans trouble des conduites. Les résultats établissent que les enfants ayant un trouble des conduites proviennent de familles moins stables et cohésives, ainsi que d'un niveau socioéconomique plus faible. De plus, les enfants ont un réseau de soutien social moins développé. L'étude révèle également que les parents des enfants qui présentent un trouble des conduites utilisent davantage la punition. Enfin, la recherche démontre que les enfants de ce groupe ont une probabilité beaucoup plus grande de présenter un autre trouble extériorisé (66 \%) que les enfants du groupe témoin $(8 \%)$. L'ensemble de ces caractéristiques distinctives permet de classifier correctement 93,8 \% des enfants de l'étude dans les 2 groupes. Les résultats obtenus indiquent l'intérêt d'améliorer les pratiques éducatives des parents, d'augmenter le réseau social de l'enfant et de réduire les conduites d'opposition et d'inattention chez l'enfant. Enfin, la complexité des difficultés relevées chez les enfants et les familles invitent à une intervention professionnelle concertée. 


\title{
Enfants manifestant un trouble des conduites et utilisant des services psychoéducatifs : un portrait social, familial et psychologique
}

\author{
Jean Toupin* \\ Robert Pauzé** \\ Éric Yergeau** \\ Michèle Déry** \\ Laurier Fortin** \\ Henri Mercier**
}

Les études contemporaines suggèrent que la plupart des enfants qui manifestent un trouble des conduites présentent également des difficultés personnelles et familiales. Ceci est particulièrement vrai pour les jeunes dont le trouble est précoce. L'identification des caractéristiques personnelles et des facteurs sociaux et familiaux de risque et de protection associés aux difficultés sont d'une grande utilité pour la planification des interventions et l'organisation des services à offrir aux jeunes et aux familles du Québec. En conséquence, les objectifs de cette étude sont d'identifier les facteurs sociaux, familiaux et psychologiques qui caractérisent les enfants présentant un trouble des conduites. Pour y parvenir 62 enfants en traitement, âgés entre 7 et 12 ans et présentant un diagnostic de trouble des conduites sont comparés à 36 enfants du même âge sans trouble des conduites. Les résultats établissent que les enfants ayant un trouble des conduites proviennent de familles moins stables et cohésives, ainsi que d'un niveau socioéconomique plus faible. De plus, les enfants ont un réseau de soutien social moins développé. L'étude révèle également que les parents des enfants qui présentent un trouble des conduites utilisent davantage la punition. Enfin, la recherche démontre que les enfants de ce groupe ont une probabilité beaucoup plus grande de présenter un autre trouble extériorisé $(66 \%)$ que les enfants du groupe témoin (8\%). L'ensemble de ces caractéristiques distinctives permet de classifier correctement 93,8 \% des enfants de l'étude dans les 2 groupes. Les résultats obtenus indiquent l'intérêt d'améliorer les pratiques éducatives des parents, d'augmenter le réseau social de l'enfant et de réduire les conduites d'opposition et d'inattention chez l'enfant. Enfin, la complexité des difficultés relevées chez les enfants et les familles invitent à une intervention professionnelle concertée.

\footnotetext{
* Professeur, Département d'éducation spécialisée, Université de Sherbrooke.
}

** Groupe de recherche sur les inadaptations sociales de l'enfance (GRISE), Université de Sherbrooke. Cette étude a bénéficié de l'appui financier du Conseil québécois de la recherche sociale (CQRS) et de l'Université de Sherbrooke. 
'identification des caractéristiques sociales, familiales et psychologiques des jeunes qui recourent à des services éducatifs et psychosociaux est importante à plus d'un titre. Elle peut permettre d'établir la présence de sous-groupes selon le type et le niveau des difficultés présentées. Elle renseigne également sur les facteurs de risque et de protection susceptibles d'influencer l'évolution des difficultés. Ces informations sont importantes pour désigner les cibles d'intervention et prévoir l'organisation des services. Cet article s'intéresse à ces aspects dans le cas des jeunes qui présentent un trouble des conduites précoce.

Le trouble des conduites désigne un ensemble de conduites antisociales répétitives qui bafouent les droits d'autrui ou les normes sociales (APA, 1987; APA, 1994). Deux enquêtes épidémiologiques canadiennes datant de quelques années révèlent une prévalence élevée du trouble des conduites chez les jeunes, variant de $1,8 \%$ à 10,4\% selon l'âge et le sexe (Offord et al., 1991 ; Valla et al., 1994a). Pour le trouble des conduites à début précoce le ratio garçon-fille s'établit à des niveaux variables selon les études, soit 2,5: 1 (Fergusson et al., 2000), 3,5: 1 (Moffitt et al., 2001), $3: 1$ (Breton et al., 1997) et 4,2: 1 (Cohen et al., 1993).

La prévalence et la stabilité temporelle du trouble (Anderson et al., 1987 ; Loeber et al., 2000; Offord et al., 1992 ; Velez et al., 1989; Verhulst et al., 1985 ; Zoccolilo et al., 1992) ont justifié un nombre substantiel de recherches. Certaines portent sur les caractéristiques sociales, familiales et psychologiques de ces jeunes à partir d'échantillons provenant de la population générale tandis que d'autres se basent sur des échantillons recevant des services. S'il nous faut reconnaître qu'il s'agit là de deux tendances de recherche, l'une plus fondamentale et l'autre appliquée, elles apportent des informations complémentaires pour l'organisation des services destinés à ces jeunes.

\section{Caractéristiques sociales, familiales et psychologiques des jeunes}

Une revue de la documentation scientifique suggère la nécessité de considérer à la fois certaines caractéristiques individuelles et du milieu social et familial pour comprendre la survenue et éventuellement, la continuation des conduites antisociales des enfants ${ }^{1}$ (Moffitt, 1993; Webster-Stratton, 1990; Yoshikawa, 1994).

L'accent de ce texte est mis sur les facteurs de risque caractérisant l'enfant, sa famille et son milieu, et qui sont potentiellement modifiables grâce à des interventions psychosociales. Conformément au modèle théorique proposé par Patterson et al. (1992), nous insistons sur les caractéristiques et le fonctionnement de la famille, les relations avec les pairs et les comportements de l'enfant. Bien que ces facteurs sont 
parfois abordés individuellement et de façon statique, ils doivent néanmoins être compris en interaction selon une perspective développementale.

Du point de vue des facteurs de risque familiaux et sociaux, les jeunes qui manifestent des conduites antisociales proviennent plus souvent de familles comportant davantage d'enfants (West et Farrington, 1973) et soumises à des conditions adverses telles que la pauvreté et la dépendance de la sécurité du revenu (Fergusson et al., 1996; Kolvin et al., 1988; Pakiz et al., 1997; Stattin et Magnusson, 1991; Werner et Smith, 1992). Les travaux de recherche indiquent également un risque accru de difficultés chez les enfants provenant de familles monoparentales (McLanahan et Booth, 1989). Il semble que cette association ne soit pas entièrement explicable par la pauvreté. Une hypothèse est que les familles monoparentales sont plus mobiles au plan résidentiel et que cette mobilité est elle-même reliée aux problèmes de comportement de l'enfant (McCormick et al., 1996). Notons également que les conflits entre conjoints sont associés aux problèmes de comportement (Reid et Crisafulli, 1990). Enfin, les difficultés de fonctionnement dans la famille et la présence de stress environnementaux et développementaux sont également associés aux difficultés des jeunes (Tolan, 1988).

Les travaux portant sur les pratiques éducatives des parents signalent des relations moins favorables entre le parent et l'enfant ayant des conduites antisociales en ce qui concerne les soins et l'affection prodigués (Kolvin et al., 1988; McCord, 1979) et certaines pratiques éducatives (McCord, 1979; Pakiz et al., 1997). Des études démontrent également une association entre la psychopathologie parentale et les conduites antisociales de l'enfant (Loeber et al., 1998; McCormick et al., 1996). Enfin, des études mentionnent un taux de criminalité plus élevé dans la famille d'origine de ces jeunes, en particulier chez le père ou des membres de la fratrie (Farrington, 1990; Farrington et West, 1993; Fergusson et al., 1996; McCord, 1979; Pakiz et al., 1997; Robins, 1979).

Des travaux complémentaires ont examiné les influences des relations avec les pairs. Ainsi, l'une des pistes de recherche a été l'étude de la contribution du rejet des pairs à la continuation des conduites antisociales. Patterson et Bank (1989) ont observé que le rejet par des pairs chez les élèves de $4^{\mathrm{e}}$ année prédisait les conduites antisociales deux ans plus tard. Des données similaires sont rapportées jusqu'à l'adolescence (Coie et al., 1995). De tels résultats permettent l'hypothèse d'un réseau social de soutien de taille inférieure chez les enfants ayant un trouble des conduites. 
Du point de vue des facteurs de risque individuels, certaines études rapportent des déficits d'attention à la tâche (Fergusson et al., 1996, 1997 ; Loeber et al., 1997), de performance intellectuelle et des fonctions «exécutives» (Kratzer et Hodgins, 1999; Moffitt et al., 1994; Stattin et Magnusson, 1991; Werner et Smith, 1992). Plusieurs études signalent que la présence d'un diagnostic additionnel à celui du trouble des conduites, en particulier celui du déficit d'attention avec hyperactivité, est un facteur de risque dans la continuation des conduites antisociales (Farrington et al., 1990; Fergusson et al., 1993; Satterfield et Schell, 1997).

Trois limites à cette recension méritent toutefois d'être soulignées. Les résultats présentés s'appliquent souvent à des échantillons composés exclusivement de garçons (Farrington et West, 1993; Loeber et al., 1977; Tremblay et al., 1994). De plus, il est utile de comprendre ces résultats en considérant simultanément plusieurs facteurs puisqu'ils sont susceptibles d'être associés les uns aux autres. Par exemple, les déficits neuropsychologiques caractérisant les jeunes avec trouble des conduites n'étaient plus associés lorsque les symptômes d'inattention étaient contrôlés statistiquement (Frost et al., 1989; Moffitt et Henry, 1989). Enfin, la plupart des résultats proviennent d'études ayant recours à un échantillon de la population générale. Il n'est pas clair que les caractéristiques des jeunes en provenance d'échantillons cliniques soient comparables. Il importe de développer brièvement ce dernier point.

\section{Utilisation des services sociaux et de santé par les jeunes}

Les enquêtes en population générale révèlent que parmi les jeunes rencontrant au moins un diagnostic de trouble mental, entre $15 \%$ et $30 \%$ d'entre eux avaient utilisé des services sociaux et de santé au cours de la dernière année (Anderson et al., 1987; Cohen et al., 1991; Offord et al., 1987; Valla et al., 1994a).

Un bon nombre des caractéristiques des familles et des jeunes précédemment décrites comme étant associées aux conduites antisociales sont également liées à une probabilité accrue de recevoir des services sociaux ou de santé. Parmi les caractéristiques familiales, le faible niveau socio-économique (Offord et al., 1991), le stress familial (Verhulst et van der Ende, 1997) et les habiletés parentales moindres (Woodward et al., 1997) sont liées à l'utilisation de services. Parmi les caractéristiques psychologiques de l'enfant, il semble que le type de difficultés présentées, soit la présence du trouble des conduites ou du trouble oppositionnel (Cohen et al., 1991), la sévérité de la symptomatologie dépressive (Compas et al., 1997), l'existence de plus d'un 
trouble (Bird et al., 1993; Breton et al., 1997) et les difficultés académiques et sociales à l'école (Verhulst et van der Ende, 1997; Woodward et al., 1997) sont corrélées à l'utilisation de services. De tels résultats suggèrent que les jeunes et les familles recevant des services présentent davantage de difficultés.

L'utilisation des services de santé mentale varie également selon le sexe. L'enquête Santé Ontario indique une prévalence plus élevée d'utilisation des services spécialisés en milieu scolaire par les garçons (Offord et al., 1987). Ceci n'est pas le cas dans l'enquête de New York (Cohen et al., 1991). Dans l'enquête québécoise (Breton et al., 1997), l'utilisation des services est particulièrement associée à la présence d'un trouble mental chez les garçons de niveau primaire.

Ces données suggèrent que les enquêtes populationnelles présentent un portrait probablement incomplet des facteurs de risque et de protection caractérisant les jeunes qui utilisent des services. Les échantillons tirés de la population générale n'incluent qu'environ $30 \%$ d'enfants ayant reçu des services de santé mentale. Ces enquêtes sont moins susceptibles d'inclure les jeunes hébergés dans des milieux spécialisés (Centres jeunesse, hôpitaux, etc.). Enfin, les recherches suggèrent que les enfants et les familles en traitement sont plus susceptibles de présenter des problèmes sévères. Le portrait d'utilisateurs de services permet de décrire les difficultés spécifiques des jeunes et des familles actuellement pris en charge, de même que d'identifier les facteurs de risque et de protection potentiels dans l'évolution des difficultés. Ces informations sont utiles pour identifier les cibles pertinentes d'intervention, réfléchir sur l'organisation des services et éventuellement mieux définir les compétences et la formation requise par le personnel intervenant.

\section{Objectifs}

Décrire les caractéristiques sociales, familiales et psychologiques d'enfants présentant des troubles des conduites et recevant des services en comparaison avec des enfants sans trouble des conduites.

Identifier parmi l'ensemble des caractéristiques celles qui discriminent les jeunes qui présentent ou non un trouble des conduites.

\section{Méthodologie}

\section{Participants}

L'échantillon comprend 62 enfants (56 garçons, 6 filles), âgés entre 7 et 12 ans ( $M=120,2$ mois), présentant tous un diagnostic de 
trouble des conduites (groupe TC) selon les critères du DSM-III-R (APA, 1987). Toutefois, certains critères ont été adaptés à l'âge des enfants afin de considérer la continuité hétérotypique des conduites antisociales (Moffitt, 1993). C'est ainsi que le symptôme de fugues nocturnes à au moins deux reprises a été modifié pour celui de rentrées tardives (trois heures après l'heure de rentrée). De même, le symptôme «a contraint quelqu'un à avoir des relations sexuelles » a été modifié pour «a initié des jeux sexuels sans le consentement de l'autre». La présence du trouble des conduites repose sur l'observation d'au moins trois symptômes au cours des 6 derniers mois selon l'intervenant qui côtoyait régulièrement l'enfant. Le taux de participation dans le groupe TC s'est établi à $62 \%$ puisque 38 parents ont refusé de participer ou n'ont pas complété l'ensemble des mesures. Les règles relatives au consentement en recherche ne permettent pas de savoir si les non-participants présentent des caractéristiques distinctives. Les enfants de l'étude bénéficiaient de services spécialisés dispensés dans des Centres de réadaptation sous forme d'hébergement $(n=12)$ ou de centre de jour $(n=24)$, ou dans des milieux scolaires $(n=26)$, ceci dans le but d'inclure dans l'échantillon divers niveaux de sévérité du trouble. Une analyse complète des facteurs personnels et familiaux associés aux divers types de prise en charge révèle beaucoup plus de ressemblances que de différences entre les sous-groupes (Toupin et al., 1998). C'est ainsi qu'ils ne se différencient sur aucune des caractéristiques familiales mesurées. La seule différence est une tendance à présenter davantage de symptômes de trouble des conduites pour les enfants en internat (moyenne $=5,3$ ) par rapport aux enfants suivis en milieu scolaire (moyenne $=4,4$ ) ou en Centre de jour (moyenne $=4,0)$ selon les intervenants $(\mathrm{F}(2,59)=7,3 ; \mathrm{p}<.01)$. Ces similitudes entre les sous-groupes justifient de les regrouper.

Un groupe de comparaison constitué d'enfants qui ne présentaient pas de troubles des conduites $(n=36)$ a également été recruté dans les mêmes écoles que les enfants qui recevaient des services en milieu scolaire. Le taux de participation dans ce groupe est de $78 \%$. Ces enfants ne présentent pas de différences quant à l'âge $(F(1,96)=0,44 ; n . s$. $)$ et au sexe $\left.X^{2}(1, \mathrm{n}=98)=1,89\right) ; n . s$. $)$ par rapport au groupe TC.

\section{Mesures}

Les enfants et le parent répondant ont été évalués à l'aide de plusieurs instruments de mesure administrés lors d'entrevues avec le parent et l'enfant ou autocomplétés. 


\section{Caractéristiques sociales et familiales}

Structure familiale

Des questions tirées du protocole de l'enquête Santé Québec sur les jeunes servent à décrire l'unité familiale (Valla et al., 1994a). En plus de déterminer dans quel type de famille vit l'enfant (intacte, monoparentale, recomposée ou d'accueil), le parent identifie le nombre de personnes composant la maisonnée, leur lien avec l'enfant ainsi que le nombre de changements de configuration familiale.

\section{Statut socioéconomique}

L'indice de statut socio-économique est basé sur le niveau de scolarité des parents, le statut d'emploi et le revenu familial annuel rapportés par le parent. L'indice comprend huit niveaux. Un score faible est associé à un statut socio-économique élevé (Toupin, 1993).

Le questionnaire Life Event Checklist (stress familial vécu par l'enfant) de Johnson et McCatcheon (1980), tiré de l'enquête québécoise (Valla et al., 1994a) recense les événements de vie significatifs que pourrait avoir vécu l'enfant depuis sa naissance. Une liste de 13 événements perturbateurs incluant les changements de la configuration familiale, le décès de proches et les agressions physiques ou sexuelles sont évalués sur une échelle en 4 points de «extrêmement dérangeant» à «pas du tout dérangeant». L'indice retenu dans l'étude est le niveau total de stress rapporté par le parent répondant.

\section{Maladie physique au foyer}

L'état de santé physique des membres de la maisonnée est évalué par une liste regroupant 30 maladies chroniques (au moins 6 mois). Pour chacune des maladies, le répondant parent indique si les membres de la famille en souffrent. Ces informations permettent d'identifier la présence d'au moins une maladie chronique vécue par un des membres du foyer (Valla et al., 1994).

Le Diagnostic Interview Schedule Self-Administered (DISSA; problèmes de santé mentale du parent) de Kovess et Fournier (1990) est un instrument qui permet d'identifier la présence de certains problèmes de santé mentale du parent répondant selon le DSMIIIR: épisode dépressif majeur, dysthymie, agoraphobie, phobie simple, trouble panique et anxiété généralisée. L'instrument documente la présence de ces troubles sur toute la vie. Le DISSA est la version abrégée du Diagnostic Interview Schedule (DIS) (Robins, 1981). Les épreuves de validation de l'instrument ont été réalisées à Montréal auprès d'une population francophone et sont concluantes. 


\section{Problème d'alcool des parents}

Les habitudes du parent reliées à l'alcool sont évaluées par des questions adressées au parent répondant. Elles établissent la fréquence de la consommation d'alcool et la présence de problèmes familiaux engendrés par cette consommation (6 derniers mois). Cette dernière variable est retenue pour l'étude (Enquête Santé Québec, 1987).

\section{Trouble mental dans la famille}

Des questions ont pour but de déceler la présence de problèmes de santé mentale dans la famille élargie. Ces questions touchent la présence de problèmes émotionnels, d'hospitalisations pour trouble mental et de tentatives de suicide. Le répondant parent indique pour chacun des problèmes le membre de la famille affligé du problème. La variable retenue est la proportion de familles dont au moins un membre présente un problème de santé mentale (Valla et al., 1994a).

L'échelle de fonctionnement de la famille (Family Adaptability and Cohesion Scales) de Olson et al., (1985) dans la version traduite par Pauzé et al. (1996) a été utilisée. Elle comprend 20 items cotés vrai/faux, complétés par le parent, divisés en deux échelles spécifiques. L'échelle d'adaptabilité évalue la capacité de la famille à adapter son fonctionnement aux changements. L'échelle de cohésion mesure le degré d'engagement entre les membres de la famille. La consistance interne de la version originale et les coefficients test-retest sont satisfaisants.

L'échelle mesurant l'attitude du parent à l'égard de l'enfant (Index of Parental Attitude, Hudson, 1982) dans la version traduite par Pauzé et al. (1996) a été employée. L'échelle comporte 25 items évalués sur une échelle en 5 points (rarement, parfois, quelquefois, la plupart du temps, tout le temps). Elle permet d'évaluer l'importance des problèmes rencontrés par le parent répondant dans sa relation avec son enfant. La cohérence interne et la stabilité de la version originale sont satisfaisantes.

\section{Questionnaire sur les comportements et attitudes des parents ((QCAP)}

Deux échelles sont complétées par le parent répondant: les soins et le soutien ( 8 items) et les pratiques parentales punitives ( 8 items). Les items sont évalués grâce à une échelle en 4 points de «la plupart du temps » à «jamais». Les soins sont définis par le soutien affectif (communication, réconfort, chaleur) et instrumental (aide l'enfant lors de tâches difficiles). Les construits des deux échelles ont été confirmés par des analyses factorielles (Bergeron et al., 1992). De plus, la consistance interne et la fidélité test-retest sont satisfaisantes (Bergeron et al., 1993). 


\section{Soutien social}

Cette échelle adaptée pour l'enquête sur la santé mentale des jeunes est composée de trois catégories d'items qui évaluent la perception du parent du soutien et de la fréquence de l'aide reçue de l'entourage. Ces questions mesurent la qualité de trois types de soutien social: le soutien émotionnel, le soutien instrumental et le soutien normatif. Chaque item est accompagné d'un choix de réponses en quatre points allant de «la plupart du temps» à «jamais». Le score ici considéré est la somme du soutien social obtenu. Les indices de saturation sont acceptables lors de l'analyse factorielle (Valla et al., 1997 ; Bouchard et Desfossés, 1989).

\section{Réseau social de l'enfant}

Ce questionnaire identifie les personnes proches de l'enfant (amis, famille nucléaire, compagnons de classe, parenté, professeurs et intervenants). Pour chaque catégorie, l'enfant doit nommer les individus significatifs dans son entourage. Le nombre de personnes de chaque catégorie atteste de la taille du réseau social du jeune (Desmarais et al., 1982).

\section{Caractéristiques psychologiques de l'enfant}

Le Diagnostic Interview Schedule for Children - $2-$ Revised (DISC-2-R) de Shaffer et al. (1993) porte sur les problèmes de santé mentale de l'enfant (Breton et al., 1998). Ce questionnaire permet d'évaluer plusieurs diagnostics d'après le DSM-III-R lors d'une entrevue avec un parent répondant. Toutes les questions concernent les comportements de l'enfant au cours de la dernière année ou des derniers 6 mois. Les diagnostics évalués sont la phobie simple, l'angoisse de séparation, l'anxiété généralisée, la dépression majeure, le déficit de l'attention avec ou sans hyperactivité, les troubles oppositionnels et le trouble des conduites. La version française du questionnaire a été développée par l'équipe de Rivière des Prairies (Bergeron et al., 1992 ; Valla et al., 1994). La fidélité test-retest, la consistance interne et les épreuves de validité de critères sont satisfaisantes (Valla et al., 1997). Cet instrument a également permis d'exclure du groupe témoin les participants qui présentaient des troubles des conduites.

\section{Procédure}

Les enfants du groupe TC ont été identifiés grâce à une grille comprenant la liste des 13 symptômes du trouble des conduites. Si l'enfant présentait le nombre de critères requis selon l'intervenant, ce dernier contactait les parents (ou le tuteur de l'enfant) pour solliciter leur parti- 
cipation à l'étude et celle de l'enfant. Dans le cas des enfants du groupe témoin, puisqu'ils provenaient des mêmes écoles, l'intervenant du milieu scolaire sollicitait également les parents et l'enfant. Le parent et l'enfant étaient rencontrés séparément par des interviewers différents. Tous les interviewers ont été formés à la passation des questionnaires. L'entrevue avec l'enfant durait environ une heure et celle avec le parent exigeait environ deux heures.

\section{Résultats}

L'analyse des résultats a d'abord porté sur l'identification des caractéristiques qui différencient les enfants avec un trouble des conduites de ceux qui n'en présentent pas. Le tableau 1 permet de constater des différences statistiquement significatives. Tout d'abord, au plan de la composition familiale, on note que $69 \%$ des enfants du groupe témoin proviennent d'une famille intacte, alors que ce n'est le cas que pour $29 \%$ des enfants présentant des troubles des conduites $\left(X^{2}(3, \mathrm{n}=98)\right.$ $=16,97 ; p<.001)$. Toutefois, il n'y a pas de différences significatives entre les deux groupes sur le nombre de personnes résidant au foyer $(F(1,96)=1,70 ; n . s)$.

Les analyses révèlent également des différences significatives entre les groupes sur le niveau socioéconomique, le niveau de stress familial et le nombre de changements familiaux. Les enfants qui présentent un trouble des conduites proviennent de milieux ayant un niveau socioéconomique en moyenne plus faible $(F(1,96)=27,06 ; \mathrm{p}<.001)$ et sont exposés à un niveau de stress plus élevé $(F(1,96)=6,45 ; \mathrm{p}<.05)$. Le tableau 1 permet également de constater qu'en moyenne les enfants du groupe TC ont vécu 5,6 changements familiaux depuis la naissance alors que la moyenne s'établit à 3,1 pour les enfants du groupe témoin $(F(1,96)=6,85 ; p<.01)$. Puisque les enfants ont en moyenne 10 ans, ceci équivaut en moyenne à un changement familial à tous les deux ans. Cette différence entre les groupes ne peut s'expliquer par la taille différente de la fratrie des deux groupes.

Le tableau 1 révèle également que les familles des deux groupes ne se différencient pas sur la présence d'une maladie physique chronique $\left(X^{2}(2, \mathrm{n}=98)=1,62 ; n . s.\right)$, d'un problème d'alcool $(0 \%$ vs $2 \%)$ ou d'un trouble mental dans la famille élargie $\left(X^{2}(2, \mathrm{n}=98)=1,31 ;\right.$ n.s. $)$. Toutefois, il importe de signaler que les groupes diffèrent significativement quant à la proportion de parents ayant présenté au cours de la vie au moins un trouble mental $\left(X^{2}(2, \mathrm{n}=98)=6,05 ; p<.05\right)$. Les résultats indiquent que près de deux tiers des parents dont l'enfant présente un trouble des conduites $(65 \%)$ ont présenté un trouble mental au cours de 
leur vie tandis que la proportion pour les parents du groupe témoin s'établit à $39 \%$.

\section{Tableau 1}

\section{Comparaison des jeunes avec ou sans trouble des conduites sur des caractéristiques sociales et familiales}

Trouble des conduites

Témoins

$(\mathrm{n}=62)$ $(\mathrm{n}=36)$

\begin{tabular}{|c|c|c|c|c|c|c|}
\hline Caractéristiques & $\%$ & $M$ & $S D$ & $\%$ & $M$ & $S D$ \\
\hline $\begin{array}{l}\text { Type de famille } \\
\text { - Intacte } \\
\text { - Monoparentale } \\
\text { - Recomposée } \\
\text { - D’accueil }\end{array}$ & $\begin{array}{r}29 \\
40 \\
23 \\
8\end{array}$ & & & $\begin{array}{l}69 * * * \\
25 \\
6\end{array}$ & & \\
\hline $\begin{array}{l}\text { Nombre de personnes } \\
\text { au foyer }\end{array}$ & & 3,8 & 1,5 & & 4,2 & 0,8 \\
\hline $\begin{array}{l}\text { Niveau } \\
\text { socioéconomique }\end{array}$ & & 6,0 & 1,4 & & $4,4 * * *$ & 1,5 \\
\hline Stress familial & & 4,7 & 5,5 & & $2,3^{*}$ & 2,7 \\
\hline $\begin{array}{l}\text { Nombre de } \\
\text { changements } \\
\text { familiaux }\end{array}$ & & 5,6 & 5,0 & & $3,1 * *$ & 3,8 \\
\hline $\begin{array}{l}\text { Difficultés familiales } \\
\text { - Maladie physique } \\
\text { chronique } \\
\text { - Trouble mental } \\
\text { (parent) } \\
\text { - Problème d'alcool } \\
\text { - Trouble mental } \\
\text { (famille élargie) }\end{array}$ & $\begin{array}{r}44 \\
65 \\
\\
0 \\
35\end{array}$ & & & $\begin{array}{l}31 \\
39 * * \\
2 \\
47\end{array}$ & & \\
\hline
\end{tabular}

$* p<.05 ; * * p<.01 ; * * * p<.001$

Les résultats se rapportant aux relations intra et extra-familiales (tableau 2) permettent de constater que la cohésion familiale $(F(1,96)$ $=10,08 ; p<.01)$ est moindre pour les familles dont un enfant présente un TC. Toutefois, les familles ne sont pas différentes au plan de l'adaptabilité $(F(1,96)=3,76 ; n . s$.$) . Les pratiques éducatives des parents répon-$ dants diffèrent selon les groupes. Les soins donnés à l'enfant sont significativement moindres $(F(1,96)=13,03 ; p<.001)$ et les pratiques éducatives plus punitives $(F(1,96)=39,35 ; p<.001)$ chez les parents ayant un enfant avec un trouble des conduites. Le tableau 2 permet éga- 
lement d'observer l'absence de différences significatives entre les groupes sur l'attitude du parent à l'égard de l'enfant $(F(1,96)=, 51 ; n$.s. $)$ et sur le soutien social obtenu par le parent pour l'enfant $(F(1,96)=, 58$; n.s.). Enfin, la taille du réseau social des enfants est similaire pour les deux groupes quant au nombre d'adultes identifiés $(F(1,96)=1,05 ;$ n.s., mais diffère quant au nombre de pairs désignés $(F(1,96)=4,12 ; p<.05)$.

Tableau 2

\section{Comparaison des jeunes avec ou sans trouble des conduites sur les relations sociales et familiales}

Trouble des conduites

$$
(\mathrm{n}=62)
$$

Témoins

$(\mathrm{n}=36)$

\begin{tabular}{|l|c|c|c|c|}
\hline Interactions & $M$ & $S D$ & \multicolumn{1}{c|}{$M$} & $S D$ \\
\hline Adaptabilité familiale & 26,6 & 5,4 & 28,4 & 2,8 \\
\hline Cohésion familiale & 36,4 & 5,1 & $39,7^{* *}$ & 4,6 \\
\hline Pratiques éducatives & 16,9 & 3,9 & $19,6^{* * *}$ & 3,2 \\
- Soins & 15,8 & 2,4 & $18,9^{* * *}$ & 2,4 \\
- Punitions & 41,6 & 17,3 & 39,3 & 12,3 \\
\hline Attitude du parent à & & & & \\
l'égard de l'enfant & 8,3 & 3,1 & 7,7 & 3,7 \\
\hline Soutien social du parent & & & & \\
\hline Taille du réseau social & & & & \\
du jeune & 6,8 & 3,3 & 7,5 & 3,0 \\
- Adultes & 4,9 & 3,5 & $6,3^{*}$ & 2,9 \\
- Jeunes & & & & \\
\hline
\end{tabular}

$* p<.05 ; * * p<.01 ; * * * p<.001$

Le tableau 3 fait état des prévalences de certains troubles mentaux selon le parent répondant. Il est possible de constater que les diagnostics spécifiques du registre des troubles intériorisés ne permettent pas de distinguer les groupes: phobie $\left(X^{2}(2, \mathrm{n}=98)=1,47 ; n . s.\right)$, angoisse de séparation $\left(X^{2}(2, \mathrm{n}=91)=, 78 ; n . s.\right)$, anxiété généralisée $\left(X^{2}(2, \mathrm{n}=78)\right.$ $=52 ; n . s$.$) , hyperanxiété \left(X^{2}(2, \mathrm{n}=98)=2,20 ; n . s\right.$. $)$, dépression $\left(X^{2}(2\right.$, $\mathrm{n}=97)=2,98 ;$ n.s. $)$ et dysthymie $\left(X^{2}(2, \mathrm{n}=97)=1,68 ;\right.$ n.s. $)$. Bien que la présence de l'un ou l'autre des troubles intériorisés tend à être plus élevée (39\% vs $22 \%$ ) chez les enfants avec un trouble des conduites comparativement aux enfants du groupe témoin, cette différence n'est pas statistiquement significative $\left(X^{2}(2, \mathrm{n}=96)=2,72 ;\right.$ n.s. $)$. Malgré tout, ce dernier résultat signale que dans le groupe TC, près de 4 enfants sur 10 manifestent un trouble intériorisé selon le parent répondant. 
Par ailleurs, le tableau 3 indique des différences significatives entre les groupes sur la prévalence des troubles extériorisés. En effet, les enfants présentant un trouble des conduites sont nettement plus susceptibles d'avoir un trouble déficitaire de l'attention avec hyperactivité $\left(X^{2}\right.$ $(2, \mathrm{n}=98)=21,32 ; p<.001)$, un trouble oppositionnel $\left(X^{2}(2, \mathrm{n}=98)\right.$ $=16,47 ; p<.001)$ ou l'un ou l'autre de ces troubles $\left(X^{2}(2, \mathrm{n}=98)\right.$ $=30,75 ; p<.001)$. Les données suggèrent qu'un enfant sur deux avec TC présente également un trouble déficitaire de l'attention avec hyperactivité et que deux enfants sur trois présentent un autre trouble extériorisé. Chez les enfants du groupe témoin les prévalences de ces troubles sont respectivement de $6 \%$ et de $8 \%$.

Tableau 3

\section{Comparaison des jeunes avec ou sans trouble des conduites sur les caractéristiques psychologiques associées (selon le parent)}

Trouble des conduites

$$
(\mathrm{n}=62)
$$

Témoins

$$
(\mathrm{n}=36)
$$

\begin{tabular}{|l|c|c|}
\hline Troubles mentaux & $\%$ & $\%$ \\
\hline Phobie & 27 & 17 \\
\hline Angoisse de séparation & 10 & 6 \\
\hline Hyperanxiété & 3 & 11 \\
\hline Anxiété généralisée & 6 & 10 \\
\hline Dépression & 0 & 8 \\
\hline Dysthymie & 3 & 10 \\
\hline Au moins un trouble intériorisé & 39 & $6^{* * * *}$ \\
\hline Hyperactivité & 52 & $3^{* * *}$ \\
\hline Opposition & 40 & $8^{* * *}$ \\
\hline Au moins un trouble extériorisé & 66 & \\
\hline
\end{tabular}

$* p<.05 ; * * p<.01 ; * * * p<.001$

Une régression logistique par étape a été retenue afin d'identifier les caractéristiques de l'enfant et de la famille qui permettent de mieux discriminer les groupes (objectif 2). Dans une première étape, toutes les variables sociales et familiales significativement associées au trouble des conduites (tableau 1) ont été introduites pour établir leur contribution dans une perspective multivariée. Les étapes subséquentes ont consisté à introduire celles relatives aux interactions sociales et familiales (tableau 2) et au profil psychologique du jeune (tableau 3). 
Tableau 4

\section{Régression logistique des prédicteurs de la présence de trouble des conduites $(n=98)$}

\begin{tabular}{|c|l|c|c|c|c|c|}
\hline Étape & \multicolumn{1}{|c|}{ Prédicteurs } & \multicolumn{1}{c|}{ B } & SE & WA LD & \multicolumn{1}{c|}{ DF } & \multicolumn{1}{c|}{ P } \\
\hline \multirow{2}{*}{1} & Famille non intacte &,- 77 &, 78 &, 99 & 1 &, 321 \\
& Niveau &,- 84 &, 32 & 7,00 & 1 &, 008 \\
& socioéconomique & & & & & \\
\hline 2 & Cohésion familiale &,- 20 &, 11 & 3,19 & 1 &, 074 \\
& Punition parentale &,- 86 &, 30 & 8,14 & 1 &, 004 \\
& Nombre de jeunes &,- 31 &, 12 & 6,05 & 1 &, 014 \\
& du réseau social & & & & & \\
\hline 3 & Trouble extériorisé & $-3,07$ &, 99 & 9,53 & 1 &, 002 \\
\hline
\end{tabular}

L'analyse finale présentée au tableau 4 révèle que 6 variables permettent de distinguer les jeunes avec TC des enfants du groupe témoin. Le type de famille (intacte vs non intacte), la cohésion familiale, le niveau socioéconomique, le recours à la punition, la taille du réseau social et la présence d'un trouble extériorisé additionnel au TC sont les facteurs qui contribuent significativement et de façon indépendante, à distinguer les deux groupes étudiés. Ces 6 variables permettent de classifier correctement 93,8\% des enfants de l'échantillon dans les groupes.

\section{Discussion}

Nos résultats suggèrent que les enfants présentant un trouble des conduites et recevant des services psychoéducatifs présentent un profil psychologique, social et familial très distinctif par rapport à des élèves sans TC du même âge. En effet, en plus de la probabilité élevée de présenter un trouble extériorisé additionnel, les enfants qui manifestent un trouble des conduites sont issus de familles moins stables et cohésives d'un niveau socioéconomique plus faible. De plus, ces enfants tendent à avoir un réseau social moins développé. Toutefois, cette dernière différence pourrait éventuellement s'expliquer par des déménagements ou des changements d'école plus fréquents. En effet, les enfants ayant un trouble des conduites changent d'école plus souvent que ceux du groupe témoin. Il importe donc de considérer la taille du réseau social de l'enfant comme résultante à la fois de la stabilité géographique de la famille et des capacités interpersonnelles de l'enfant. L'étude révèle également que les pratiques éducatives des parents d'enfants TC sont davantage punitives. Il faut ici se rappeler que ces informations sont données par les parents eux-mêmes. L'interprétation des résultats doit toutefois tenir 
compte du rôle possible des troubles de comportement de l'enfant. On peut interpréter ces résultats dans la perspective où les conduites antisociales et d'opposition peuvent susciter davantage de comportements punitifs de la part des parents. Néanmoins, en reconnaissant la nature transactionnelle de la relation parent-enfant, il paraît évident que le recours fréquent à la punition est peu susceptible de favoriser une relation positive entre les parents et l'enfant. Rappelons que l'ensemble des résultats s'appliquent aux garçons, compte tenu du petit nombre de filles dans cette étude.

Plusieurs de ces résultats sont cohérents avec d'autres travaux de recherche. Le taux élevé de concomitance entre le trouble des conduites et le trouble déficitaire d'attention avec hyperactivité est souvent documenté (Lynam, 1996). Nombre de travaux suggèrent que la présence d'un déficit d'attention avec hyperactivité est associée à la continuation des conduites antisociales à l'adolescence et à l'âge adulte (Farrington et al., 1990; af Klinteberg et al., 1993; Lahey et Loeber, 1997, Loeber et al., 1990; Loeber et al., 1997; Moffitt, 1990 ; Taylor et al., 1996).

Les résultats obtenus quant aux conditions sociales et familiales rejoignent ceux d'autres études. Les jeunes manifestant des conduites antisociales sont plus susceptibles de provenir de milieux socioéconomiques moins favorisés (Fergusson et al., 1996; Pakiz et al., 1997 ; Stattin et Magnusson, 1991 ; Werner et Smith, 1992). Il est également courant de retrouver des associations entre les conduites antisociales et les pratiques éducatives des parents (Loeber et StouthamerLoeber, 1986). C'est ainsi que les soins et l'affection, de même que les pratiques éducatives seraient plus déficitaires dans ces familles (Kolvin et al., 1988; McCord, 1979; Pakiz et al., 1997).

Le ratio garçon-fille pour le trouble des conduites s'établit à environ 10:1 dans cette étude. Ce ratio diffère passablement de celui observé dans les enquêtes populationnelles soit environ 3.5:1 (Cohen et al., 1993 ; Moffitt et al., 2001 ; Breton et al., 1997). Cet écart pourrait s'expliquer par la tendance plus marquée à solliciter des services en milieu scolaire pour les garçons de niveau primaire (Breton et al., 1997; Offord et al., 1987). Si cette interprétation est exacte, les jeunes filles seraient victimes d'une double discrimination quant à l'accès aux services. D'une part, la prévalence du trouble des conduites est inférieure chez les jeunes filles alors que ce type de difficultés conduit plus souvent à la référence à des services spécialisés. D'autre part, même en présence d'un trouble des conduites, il semble y avoir une moins grande propension à référer les jeunes filles vers certains types de services. 
Les résultats de cette étude établissent l'importance d'interventions visant la réduction des difficultés familiales, l'amélioration des pratiques éducatives des parents, le renforcement du réseau social de l'enfant et la diminution des conduites d'opposition, d'inattention, en plus des conduites antisociales.

Du point de vue de l'organisation des services, la présence de sous-groupes distincts parmi les jeunes et familles référés doit également être considérée. On peut se demander s'il est possible d'intervenir dans le même contexte et avec les mêmes moyens lorsque l'enfant provient d'une famille qui dispose de très peu de ressources (santé mentale, pauvreté, instabilité). De même, il est probable que les enfants combinant le trouble des conduites et le déficit d'attention avec hyperactivité ne nécessitent pas les mêmes services que les enfants présentant seulement un trouble des conduites.

L'ampleur des difficultés observées chez ces enfants et dans les familles suggère d'autres réflexions quant à l'organisation des services. La complexité des difficultés de l'enfant et dans la famille suggère une solide formation professionnelle de niveau universitaire. La prévalence élevée de troubles mentaux parmi les parents répondants indique également l'importance que les intervenants puissent évaluer, comprendre et intervenir en tenant compte des difficultés des parents. De plus, les interventions auprès de la famille afin d'augmenter la cohésion, le renforcement des compétences parentales et l'amélioration des habiletés à s'adapter aux stresseurs et aux troubles de l'enfant nécessitent des compétences spécifiques. Les intervenants doivent être dotés d'un ensemble de connaissances solides et bénéficier d'un soutien professionnel en cours d'intervention. Les résultats suggèrent également l'importance potentielle d'un travail d'équipe coordonné impliquant plusieurs secteurs (scolaire, CLSC, Centres jeunesse, etc.).

Les résultats de cette étude doivent être interprétés avec les limites de la méthodologie utilisée. D'une part, ils présentent le portrait des enfants et des familles qui ont accepté de participer à l'étude. Certains aspects pourraient les distinguer des non-participants qui malgré tout reçoivent des services psychoéducatifs. D'autre part, les analyses portent sur de multiples comparaisons sans ajustements statistiques. Ceci rend plus probable la détection de différences entre les groupes. Enfin, pour plusieurs des mesures utilisées le répondant est le parent. Le recours à d'autres répondants (enfant, enseignant) aurait pu produire d'autres résultats.

Des travaux de recherche subséquents seraient utiles afin de suivre le développement social, familial et scolaire de ces enfants. Les trajec- 
toires de développement des enfants devraient être analysées en considérant l'ampleur des difficultés de départ et des interventions pour les résorber. De plus, il serait pertinent de mieux connaître les différences dans la nature et l'ampleur des difficultés sociales, familiales et psychologiques chez les jeunes ayant un trouble des conduites selon qu'ils bénéficient d'interventions ou au contraire sont issus de la population générale et sont sans services. De tels résultats permettraient d'établir jusqu'à quel point les résultats des enquêtes populationnelles sont valables pour les jeunes et les familles faisant l'objet d'interventions spécifiques. Enfin, il serait important d'examiner les différences et les similarités dans les facteurs de risque et de protection selon le sexe. Jusqu'à présent l'écart très significatif des prévalences et de l'utilisation des services est loin d'être expliqué.

\section{Note}

1. Nous recourons ici au terme conduites antisociales pour désigner les manifestations du trouble des conduites et les actes délinquants. Ce terme est employé parce qu'il est plus inclusif que celui de trouble des conduites. La plupart des études présentées ont porté sur la présence de conduites antisociales et délinquantes sans pour autant exiger l'atteinte du seuil du trouble des conduites.

\section{Références}

ASSOCIATION pSyChIATRIQUe AMÉRICAINE, 1987, Diagnostic and Statistical Manual of Mental Disorders, 3th ed., Washington, DC.

AsSOCIATION PSYCHIATRIQUe AmÉRICAINE, 1994, Diagnostic and Statistical Manual of Mental Disorders, 4th ed., Washington, DC.

Anderson, J.C., Williams, S., McGee, R., Silva, P.A., 1987, DSM- III disorders in preadolescent children: Prevalence in a large sample from the general population, Archives of General Psychiatry, 44, 69-76.

Bergeron, L., Valla, J. P., Breton, J. J., 1992, Pilot study for the Quebec child mental health survey: Part I. Measurement of prevalence estimates among 6 to 14 year old, Canadian Journal of Psychiatry, 3, 6, 374-380.

Bergeron, L., Valla, J. P., Breton, J. J., Gaudet, N., Berthiaume, C., 1993, Factor Analysis and Reliability of Parental Behavior and Attitude Questionnaire, Présentation au $40^{\circ}$ Congrès annuel de l'American Academy of Child and Adolescent Psychiatry, San Antonio, Texas.

Bird, H. R., Gould, M. S., Staghezza, B., 1993, Patterns of diagnostic comorbidity in a community sample of children aged 9 through 16 years, Journal of American Academy of Child Adolescent Psychiatry, 32, 2, 361-368. 
Bouchard, C., DesFossés, E., 1989, Utilisation des comportements coercitifs envers les enfants: stress, conflits et manque de soutien dans la vie des mères, Apprentissage et Socialisation, 12, 1, 19-28.

Boyle, M. H., OfFord, D. R., 1988, Prevalence of childhood disorder, perceived need for help, family dysfunction and resource allocation for child welfare and children's mental health services in Ontario, Canadian Journal of Behavioral Sciences, 20, 4, 374-387.

Breton, J.-J., Bergeron, L., Valla, J.-P., Berthiaume, C., St-Georges, M., 1998, Diagnostic interview schedule for children (DISC-2.25) in Quebec: Reliability findings in light of the MECA study, Journal of the American Academy of Child \& Adolescent Psychiatry, 37, 11, 1167-1174.

Breton, J.-J., Valla, J.-P., Bergeron, L., Berthiaume, C., Gaudet, N., Toupin, J., Blais, R., Lambert, J., St-Georges, M., Daveluy, C., 1997, Enquête québécoise sur la santé mentale des jeunes de 6 à 14 ans: Volume 2, partie 2: Utilisation des services, Rapport de recherche, Dépôt légal — Bibliothèque nationale du Québec. ISBN : 29803587 9-7.

CAPaldi, D. M., Stoolmiller, M., 1999, Co-occurrence of conduct problems and depressive symptoms in early adolescent boys: III. Prediction to young-adult adjustment, Development and Psychopathology, 11, 59-84.

Cohen, P., Cohen, J., Kasen, S., Velez, C. N., Hartmark, C., Johnson, J., Rojas, M., BROOK, J., StRuening, E. L., 1993, An epidemiological study of disorders in late childhood and adolescence - I age and gender - specific prevalence, Journal of Child Psychology and Psychiatry 34, 6, 851-867.

Cohen, P., Kasen, S., Brook, J. S., Struening, E. L., 1991, Diagnostic predictors of treatment patterns in a cohort of adolescents, Journal of American Academy of Child Adolescent Psychiatry, 30, 6, 989-993.

Coie, J. D., Terry, R., Lenox, K., Lochman, J., Hyman, C., 1995, Childhood peer rejection and aggression as predictors of stable patterns of adolescent disorders, Development and Psychopathology, 7, 697-713.

Compas, B. E., Oppedisano, G., Connor, J. K., Gerhardt, C. A., Henden, B. R., ACHENBACH, T. M., 1997, Gender differences in depressive symptoms in adolescence: Comparison of national samples of clinical referred and nonreferred youths, Journal of Consulting and Clinical Psychology, 65, 4, 617-626.

Desmarais, D., Blanchet, L., Mayer, R., 1982, Modèle d'intervention en réseau au Québec, Cahiers critiques de thérapie familiale et de pratiques de réseaux, 4-5, 109-118.

FARRINGTON, D. P., 1990, Implications of criminal career research for the prevention of offending, Journal of Adolescence, 13, 93-113 
FARRINGTON, D.P., LOEBER, R., VAN KAMMEN, W. B., 1990, Long-term criminal outcomes of hyperactivity-impulsivity-attention deficit and conduct problems in childhood, in Robins, L.N. and Rutter, M. eds., Straight and Devious Pathways from Childhood to Adulthood, Cambridge University Press, 62-81.

FARRINGTON, D. P., WeSt, D. J., 1993, Criminal, penal and life histories of chronic offenders : risk and protective factors and early identification, Criminal Behaviour and Mental Health, 3, 492-523.

Fergusson, D. M. Horwood, L. J., Linskey, M. T., 1993, The effects of conduct disorder and attention deficit in middle childhood on offending and scholastic ability at age 13, Journal of Child Psychology and Psychiatry, 34, 6, 899-916.

Fergusson, D. M., Lynskey, M. T., Horwood, L. J., 1996, Factors associated with continuity and changes in disruptive behavior patterns between childhood and adolescence, Journal of Abnormal Child Psychology, 24, 533-553.

Fergusson, D. M., Lynskey, M. T., Horwood, L. J., 1997, Attentional difficulties in middle childhood and psychosocial outcomes in young adulthood, Journal of Child Psychology and Psychiatry, 38, 633-644.

Frost, L. A., Moffitt, T. E., McGeE, R., 1989, Neuropsychological correlates of psychopathology in an unselected cohort of young adolescents, Journal of Abnormal Psychology, 98, 307-313.

Goodman, S. H., Lahey, B. B., Fielding, B., Dulcan, M., Narrow, W., REGIER, D., 1997, Representativeness of clinical samples of youths with mental disorders: a preliminary population-based study, Journal of Abnormal Psychology, 106, 1, 3-14.

Hudson, W. W., 1982, The Clinical Measurement Package: A Filed Manual, Illinois, Dorsey Press.

Johnson, J. H., McCATCheON, S. M., 1980, Assessing life stress in older children and adolescents : Preliminary findings with the Life Event Checklist, in Sarason, I.G., Spieldberger, C.D., eds., Stress and Anxiety, Washington, D.C., Emisphere.

af Klinteberg, B., Andersson, T., Magnusson, D., Stattin, I., 1993, Hyperactive behavior in childhood as related to subsequent alcohol problems and violent offending: A longitudinal study of male subjects, Personality and Individual Difference, 15, 381-388.

Kolvin, L., Miller, J. W., FleEting, J., Kolvin, P. A., 1988, Social and parenting factors affecting criminal-offence rates: Findings from the newcastle 
thousand family study (1947-1980), British Journal of Psychiatry, 152, $80-90$.

Kovess, V., Fournier, L., 1990, The DISSA: an abridged self-administered version of the DIS. Approach by episode, Social Psychiatry and Psychiatric Epidemiology, 25, 179-186.

Kratzer, L., Hodgins, S., 1999, Adult outcomes of child conduct problems : a cohort study, Journal of Abnormal Child Psychology, 25, 1, 65-81.

LAHEY, B. B., LOEBER, R., 1997, Attention-deficit/hyperactivity disorder, oppositional defiant disorder, conduct disorder, and adult antisocial behavior : A life span perspective in Stoff, D. M., Breiling, J., Maser, J. D., eds., Handbook of Antisocial Behavior, New York, Wiley.

LAROCHE, I., TouPin, J., 1998, Recension des interventions psychosociales destinées aux enfants et aux adolescents manifestant des troubles oppositionnels et des conduites, Revue québécoise de psychologie, 19, 1,1-16.

Loeber, R., Farrington, D.P., Stouthammer-Loeber, M., Van Kammen, W. B., 1998, Antisocial Behavior and Mental Health Problems : Explanatory Factors in Childhood and Adolescence, Mahwah, N. J., Laurence Erlbaum.

Loeber, R., Green, S. M., Lahey, B. B., Frick, P. J., McBurnett, K., 2000, Findings on Disruptive Behavior Disorders from the First Decade of the Developmental trends Studies, Clinical Child and Family Psychology Review, 3, 1, 37-60.

Loeber, R., Green, S., Lahey, B., Stouthamer-Loeber, M., 1990, Optimal informants on childhood disruptive behaviors, Development and Psychopathology, 1, 317-337.

Loeber, R., Keenan, K., Zhang, Q., 1997, Boys'experimentation and persistence in developmental pathways toward serious delinquency, Journal of Child and Family Studies, 6, 321-357.

Loeber, R., Stouthamer-Loeber, M., 1986, Family factors as correlates and predictors of juvenile conduct problems and delinquency, in Tonry, M., Morris, N., eds., Crime and Justice: An Annual Review of Research, 7, Chicago, University of Chicago Press, 29-149.

LyNAM, D. R., 1996, Early identification of chronic offenders: Who is the Fledgling psychopath ? Psychological Bulletin, 20, 209-234.

McCord, J., 1979, Some child-rearing antecedents of criminal behavior in adult men, Journal of Personality and Social Psychology, 37, 1477-1486.

McCormick, M., Workman-Daniels, K., Brooks-Gunn, J., 1996, The behavioural and emotional well-being of school-age children with different birth weights, Pediatrics, 97, 18-25. 
McLanahan, S., Booth, K., 1989, Mother-only families : Problems, prospects and politics, Journal of Marriage and the Family, 51, 557-580.

MofFITT, T. E., 1990, Juvenile delinquency and attention deficit disorder : boys' development trajectories from age 3 to age 15, Child Development, 61, 893-910.

MoffitT, T. E., 1993, Adolescence-limited and life-course-persistent antisocial behavior: A developmental taxonomy, Psychological Review, 100, 674701.

Moffitt, T. E., Capsi, A., RutTer, M., Silva, P. A., 2001, Sex Differences in Antisocial Behavior, Cambridge University Press.

Moffitt, T .E., Henry, B., 1989, Neuropsychological assessment of executive functions in self-reported delinquents, Development and Psychopatho$\log y, 1,105-118$.

Moffitt, T. E., LynAm, D. R., SiLvA, P. A., 1994, Neuropsychological tests predicting persistent male delinquency, Criminology, 32, 277-300

Offord, D. R., Boyle, M. C., Racine, Y. A., 1991, The epidemiology of antisocial behavior in childhood and adolescence, in Pepler, D .J. and Rubin, K. H., eds., The Development and Treatment of Childhood Aggression, Hilsdale, N.J., Erlbaum, 31-54.

Offord, D. R., Boyle, M. H., Racine, Y., Fleming, J. E., Cadman, D.T., Munroe Blum, H., Byrne, C., Links, P. S., Lipman, E. L., MacMillan, H. L., Rae-Grant, N., Sanford, M., Szatmari, P., Thomas, H., WoodWARD, C.A., 1992, Outcome, prognosis and risk in a longitudinal followup study, Journal of the American Academy of Child an Adolescent Psychiatry, 31, 916-923.

Offord, D. R., Boyle, M. H., Szatmari, P., Rae-Grant, N. I., Links, P. S., Cadman, D. T., Byles, J. A., Crawford, J.W., Munroe Blum, H., Byrne, C., Thomas, H., Woodward, C. A., 1987, Ontario child health study, Archives of General Psychiatry, 44, 832-836.

Olson, D. H., Portner, J. LAVER, Y., 1985, FACES III, Family Social Science, University of Minnesota.

PAKiz, B., Reinherz, H., GiaconiA, R. M., 1997, Early risk factors for serious antisocial behavior at age 21 : A longitudinal community study, American Journal of Orthopsychiatry, 67, 92-100.

PAtTerson, G. R., BANK, L., 1989, Some amplifying mechanisms for pathologic processes in families, in Gunnar, M., Thelen, E., eds., Symposia on Child Psychology, Hillsdale, NJ : Laurence Erlbaum, 167-320. 
Patterson, G. R., Reid, J. B., Dishion, T .J., 1992, Antisocial Boys : A Social Interactional Approach, Eugene, OR, Castalia.

Pauzé, R., Toupin, J., DÉRY, M., HotTe, J-P., 1996, Étude des caractéristiques sociofamiliales et personnelles associées au placement d'enfants en Centre de réadaptation pour jeunes en difficulté d'adaptation dans la région de Montréal, Rapport de recherche soumis au CRSSSMM.

REID, W. J., CRISAFUlLI, A., 1990, Marital discord and child behavior problems : A meta-analysis, Journal of Abnormal Child Psychology, 18, 105-117.

RoBINs, L. N., 1979, Study chilhood predictors of adult outcomes : Replications from longitudinal studies, in Barrett, J. E., Rose, R. M., Klerman, G. L., eds., Stress and Mental Disorder, New York, Raven Press.

RoBINS, L. N., 1981, Epidemiological approaches to natural history research: Antisocial disorders in children, Journal of the American Academy of Child and Adolescent Psychiatry, 20, 3, 566-580.

Robins, L. N., RATCLIFF, K. S., 1979, Risk factors in the continuation of childhood antisocial behavior into adulthood, International Journal of Mental Health, 7, 96-116.

SATterfield, J. H., Schell, A., 1997, A prospective study of hyperactive boys with conduct problems andnormal boys: Adolescent and adult criminality, Journal of American Academy of Child and Adolescent Psychiatry, $36,12,1726-1735$.

Shaffer, D., Schwab-Stone, M., Fisher, P., Cohen, P., Piacentini, J., Davies, M., Conners, C.K., Regier, D., 1993, The diagnostic interview for children-revised version (DISC-R) : I. Preparation, field testing, interrator reliability, and acceptability, Journal of the American Academy of the Child and Adolescent Psychiatry, 32, 643-650.

SiEgelman, M., 1965, Evaluation of Bronfenbrenneer's questionnaire for children concerning parental behavior, Child Development, 36, 163-174.

Stattin, H., Magnusson, D., 1991, Stability and change in criminal behaviour up to age 30, British Journal of Criminology, 31, 4, 327-346.

Taylor, E., Chadwick, O., Heptinstall, E., Danckaerts, M., 1996, Hyperactivity and conduct problems as risk factors for adolescent development, Journal of American Academy of Child and Adolescent Psychiatry, 35, 9, 1213-1226.

Tolan, P., 1988, Socioeconomic, family and social stress correlates of adolescent antisocial and delinquent behavior, Journal of Abnormal Child Psychology, 16, 3, 317-331. 
ToupIN, J., 1993, Échelle de statut socio-économique, Université de Sherbrooke.

Toupin, J., DÉry, M., Fortin, L., Mercier, H., PAuZÉ, R., 1998, Caractéristiques sociales, familiales et psychologiques manifestant des troubles des conduites, Rapport final présenté au Conseil Québécois de la recherche sociale.

Tremblay, R. E., Pihl, R. O., Vitaro, F., Dobkin, P. L., 1994, Predicting early onset of male antisocial behavior from preschool behavior, Archives of General Psychiatry, 51, 732-739.

Valla, J. P., Bergeron, L., Bérubé, H., Gaudet, N., St-Georges, M., 1994, A structured pictorial questionnaire to access DSM-III-based diagnoses in children (6-11 years): Development, validity, and reliability, Journal of Abnormal Child Psychology, 22, 4, 403-423.

Valla, J.-P., Breton, J.L., Bergeron, L. Gaudet, N., Berthiaume, C., SaintGeorges, M., Daveluy, C., Tremblay, V., Lambert, J., Houde, L., LÉPINE, S., 1994a, Enquête québécoise sur la santé mentale des jeunes de 6 à 14 ans, Rapport synthèse, Hôpital Rivières-des-Prairies et Santé Québec.

Valla, J.-P., Bergeron, L., Breton, J. J., Gaudet, N., Berthiaume, C., StGeorges, M., Daveluy, C., Tremblay, V., Lambert, J., Houde, L., LÉPINE, S., SMOLla, N., 1997, Enquête québécoise sur la santé mentale des jeunes de 6 à 14 ans 1992, Volume 1, Méthodologie, Rapport de recherche.

Velez, C.N., Johnson, J., Cohen, P., 1989, Longitudinal analyses of selected risk factors for childhood psychopathology, Journal of the American Academy of Child and Adolescent Psychiatry, 28, 861-864.

Verhulst, F. C., Berden, F. G. SAnders-Woudstra, J. A., 1985, Mental health in Dutch children: II. The prevalence of psychiatric disorders and relationship between measures, Acta Psychiatrica Scandinavia, 72, (suppl. 324), 1-45.

Verhulst, F. C., van der Ende, J., 1997, Factors associated with child mental health service use in the community, Journal of American Academy of Child Adolescent Psychiatry, 36, 7, 901-909.

Webster-Stratton, C., 1990, Stress : A potential disruptor of parent perceptions and family interactions, Journal of Clinical Child Psychology, 19, 4, 302-312.

Werner, E. E., SMith, R. S., 1992, Overcoming the Odds : High Risk Children from Birth to Adulthood, New York, Cornell University Press. 
West, D. J., Farrington, D. P., 1973, Who Becomes Delinquent? London, Heinemann.

Woodward, L., Dowdney, L., TAYlor, E., 1997, Child and family factors influencing the clinical referral of children with hyperactivity : A research note, Journal of Psychology and Psychiatry, 38, 4, 479-485.

YoshiKawa, H., 1994, Prevention as cumulative protection: effects of early family support and education chronic delinquency and its risks, Psychological Bulletin, 115, 28-54.

Zoccolilo, M., Pickles, A., Quinton, D., RutTer, M., 1992, The outcome of childhood conduct disorder: Implications for defining adult personality disorder and conduct disorder, Psychological Medicine, 22, 971-986.

\section{ABSTRACT}

\section{Children with conducts disorder and using psychoeducational services: a social, psychological and family portrait}

Contemporary studies suggest that most of the children who manifest conducts disorder also present personal as well as family problems. This is particularly true for children whose disorder is precocious. The identification of personal characteristics as well as social and family risk and protection factors associated with children with such problems are of great use for intervention planning and service organization for them and their families in Québec. In consequence, the objectives of this study are to identify the social, family and psychological factors that characterize children presenting conducts disorder. To do so, 62 children in treatment between the ages of 7 and 12 and with a diagnosis of conducts disorder are compared with 36 children of the same age without such diagnosis. Results show that children with conduct problems come from less stable and cohesive families as well as a lower socio-economic level. Moreover, their network of social support is less developed. The study reveals that the parents of children with conducts disorder use punishment more often. Finally, the research demonstrates that the children of this group have a much higher probability of presenting an exteriorized disorder (66\%) than the children of the other group (8\%). All these distinctive characteristics allows to correctly classify $93,8 \%$ of the children of this study in the 2 groups. The results indicate the need to improve the parents'educational practices, increase the child's social network as well as reduce the conducts of opposition and inattention of the child. Finally, the complexity of the problems raised with the child and families commands a concerted professional intervention. 


\section{RESUMEN}

Los niños que manifiestan un problema de conducta y utilizan los servicios psicoeducativos: un retrato social, familiar y psicológico

Los estudios contemporáneos sugieren que la mayoría de los niños que manifiestan un problema de conducta presentan también dificultades personales y familiares. Esto es particularmente cierto en los jóvenes cuyo problema es precoz. La identificación de las características personales y los factores sociales y familiares de riesgo y protección asociados a los niños con dichas dificultades son de gran utilidad para la planificación de las intervenciones y la organización de servicios por ofrecer a los jóvenes y a las familias de Quebec. En consecuencia, los objetivos de este estudio son identificar los factores sociales, familiares y psicológicos que caracterizan a los niños que presentan un problema de conducta. Para lograrlo, 62 niños en tratamiento, entre 7 y 12 años y con un diagnóstico de problemas de conducta, son comparados con 36 niños de la misma edad sin problemas de conducta. Los resultados establecen que los niños que tienen un problema de conducta provienen de familias menos estables y cohesivas, así como de un nivel socioeconómico más débil. Además, los niños cuentan con una red de apoyo social menos desorrollada. El estudio también revela que los padres de los niños que presentan un problema de conducta utilizan más el castigo. Por último, la investigación demuestra que los niños de este grupo tienen una probablidad mucho mayor de presentar otro problema exteriorizado (66\%) que los niños del grupo testigo (8\%). El conjunto de estas características distintivas permite clasificar correctamente al 93.8\% de los niños del estudio en los 2 grupos. Los resultados obtenidos indican el interés de mejorar las prácticas educativas de los padres, aumentar la red social del niño y reducir las conductas de oposición y distracción en el niño. Finalmente, la complejidad de las dificultades observadas en los niños y las familias invitan a una intervención profesional adecuada.

\section{RESUMO}

\section{Retrato social, familiar e psicológico das crianças que manifestam problemas de comportamento e utilizam serviços psico-educativos}

Estudos contemporâneos afirmam que a maioria das crianças que manifesta problemas de comportamento apresenta também dificuldades pessoais e familiares. Principalmente jovens cujo problema aparece bem cedo. A identificação das características pessoais e dos fatores sociais e familiares de risco e de proteção, associados às crianças com tais 
dificuldades, é de grande utilidade para o planejamento das atuações e para a organização dos serviços a oferecer aos jovens e às famílias do Quebec. Conseqüentemente, os objetivos deste estudo foram identificar os fatores sociais, familiares e psicológicos que caracterizam as crianças que apresentam problemas de comportamento. Para tal, 62 crianças em tratamento, de 7 à 12 anos e apresentando um diagnóstico de problemas de comportamento, foram comparadas a 36 crianças da mesma idade, sem problemas de comportamento. Os resultados demonstraram que as crianças que apresentam problemas de comportamento vêm de famílias menos estáveis e unidas, e de um nível sócio-econômico mais baixo. Além disto, as crianças possuem uma rede de apoio social menos desenvolvida. $\mathrm{O}$ estudo revela também que os pais das crianças que apresentam problemas de comportamento utilizam mais a punição que os outros pais. Concluindo, a pesquisa prova que as crianças desse grupo têm uma probabilidade muito maior de apresentar outros problemas exteriorizados (66\%) que as crianças do grupo de controle (8\%). Essas características distintivas permitem classificar corretamente $93,8 \%$ das crianças do estudo nos dois grupos. Os resultados obtidos indicam o interesse em melhorar as práticas educativas dos pais, aumentar a rede social da criança e reduzir os comportamentos de oposição e de falta de atenção na criança. A complexidade das dificuldades levantadas junto às crianças e às famílias incentiva a preparação de uma atuação profissional. 